\title{
gु \\ Evidence for triplet superconductivity in Josephson junctions with barriers of the ferromagnetic Heusler alloy $\mathrm{Cu}_{2} \mathrm{MnAl}$
}

\author{
D. Sprungmann, ${ }^{1}$ K. Westerholt, ${ }^{1}$ H. Zabel, ${ }^{1}$ M. Weides,${ }^{2}$ and H. Kohlstedt ${ }^{3}$ \\ ${ }^{1}$ Institut für Experimentalphysik/Festkörperphysik, Ruhr-Universität Bochum, 44780 Bochum, Germany \\ ${ }^{2}$ Physics Department, Broida Hall, UC Santa Barbara, California 93106, USA \\ ${ }^{3}$ Nanoelektronik, Technische Fakultät Kiel, Christian-Albrechts-Universität Kiel, Kiel 24143, Germany
}

(Received 21 July 2010; published 9 August 2010)

\begin{abstract}
We have studied Josephson junctions with barriers prepared from the Heusler compound $\mathrm{Cu}_{2} \mathrm{MnAl}$. In the as-prepared state the $\mathrm{Cu}_{2} \mathrm{MnAl}$ layers are nonferromagnetic and the critical Josephson current density $j_{c}$ decreases exponentially with the thickness of the Heusler layers $d_{F}$. On annealing the junctions at $240{ }^{\circ} \mathrm{C}$ the Heusler layers develop ferromagnetic order and we observe a dependence $j_{c}\left(d_{F}\right)$ with $j_{c}$ strongly enhanced and weakly thickness dependent in the thickness range $7.0<d_{F}<10.6 \mathrm{~nm}$. We interpret this feature as an indication of a triplet component in the superconducting pairing function generated by the specific magnetization profile inside thin $\mathrm{Cu}_{2} \mathrm{MnAl}$ layers.
\end{abstract}

DOI: 10.1103/PhysRevB.82.060505

PACS number(s): 74.50.+r, 74.45.+c, 75.70.Cn

Superconducting pairing functions with a symmetry different from conventional $s$-wave singlet pairing are in the focus of interest since the advent of BCS theory. ${ }^{1}$ However, unconventional pairing functions are rare in nature and experimental realizations had to wait until detecting systems such as the heavy fermion, ${ }^{2}$ the high- $T_{c}{ }^{3}$ and the $\mathrm{Sr}_{2} \mathrm{RuO}_{4}$ superconductors. ${ }^{4}$ Unconventional pairing states might also be induced by the superconducting proximity effect at superconducting/ferromagnetic (S/F) interfaces (see Refs. 5 and 6 for recent reviews). The exchange field in the ferromagnetic layer favors triplet pairing, i.e., a superconducting condensate function with parallel spins. The penetration depth of superconducting triplet pairs into a ferromagnet in the limit of dirty metals with weak pair breaking scattering is given by $\xi=\sqrt{\hbar D_{F} / 2 \pi k_{B} T}$ (electron-diffusion constant $D_{F}$ ) whereas the penetration depth of singlet pairs is limited by the ferromagnetic exchange energy $E_{e x}$ via $\xi_{F}=\sqrt{\hbar D_{F} / E_{e x}} .5$ Thus at low temperatures triplet pairs can penetrate deeply into a ferromagnetic metal.

Measurements on lateral Josephson junctions with half metallic $\mathrm{CrO}_{2}$ (Ref. 7) and the rare-earth metal Ho (Ref. 8) as the barrier material gave indications of very weak longrange triplet contributions to the supercurrent. In vertical Josephson junctions (with the current direction perpendicular to the layers) with ferromagnetic barrier layers of variable thickness $d_{F}$ the decay of the superconducting critical current $j_{c}\left(d_{F}\right)$ directly defines the superconducting decay length. Up to now, however, the experimental studies of $j_{c}\left(d_{F}\right)$ with barriers made from elemental ferromagnetic transition metals or alloys did not indicate long-range triplet superconductivity. ${ }^{9}$ Singlet pairing with a transition from 0 to $\pi$ coupling ( 0 and $\pi$ denoting the phase shift of the pair wave function across the barrier) could reasonably well explain all data.

Theoretical work suggested an interesting possibility to enhance the amplitude of the triplet component at the S/F interface drastically. Instead of a homogeneous magnetization profile one should better use a ferromagnetic layer system with some intrinsic spin canting, e.g., an in-plane domain wall at the interface, ${ }^{10}$ an $\mathrm{S} / \mathrm{F} / \mathrm{S}$ multilayer with nonparallel orientation of the $\mathrm{F}$ layers, ${ }^{11}$ or a trilayer system with canted spins on both sides facing the S layers. ${ }^{12}$ In these model calculations the triplet component in the condensate function is enhanced by conversion of singlet Cooper pairs into triplet pairs by the spin active interfaces. ${ }^{13}$ The dominating superconducting wave function is the so-called oddfrequency triplet pairing, i.e., a superconducting wave function even in space, even in spin but odd in time (or odd in the Matsubara frequencies). This exotic type of pairing state has been proposed originally for the pairing in the ${ }^{3} \mathrm{He}$ superfluid. ${ }^{14}$ Following these ideas Khaire et al. ${ }^{15}$ very recently incorporated a complicated ferromagnetic multilayer structure with separate weakly magnetic interface layers and a Co-core layer as a barrier in Josephson junctions and could demonstrate a weakly decaying $j_{c}\left(d_{F}\right)$ in favor odd triplet superconductivity.

In this Rapid Communication we will show that with a ferromagnetic Heusler alloy $\mathrm{Cu}_{2} \mathrm{MnAl}$ as the barrier one can create a magnetization profile which also seems to be very effective in generating triplet superconductivity. Our Josephson junctions with a lateral size of $10 \times 50$ or 10 $\times 200 \mu \mathrm{m}^{2}$ were grown by magnetron sputtering and microstructured by a combination of optical lithography and ionbeam etching, as described in detail in Ref. 16. The barrier between the two $\mathrm{Nb}$ electrodes is composed of an about 1-nm-thick $\mathrm{AlO}_{x}$ layer and a $\mathrm{Cu}_{2} \mathrm{MnAl}$ layer. The thin $\mathrm{AlO}_{x}$ layer enhances the normal resistance $R_{n}$ without changing the physics discussed above. In a single preparation run we prepared several hundred junctions covering the thickness range from typically 5 to $15 \mathrm{~nm}$ for the Heusler alloy while keeping the other layer thicknesses constant.

The magnetization measurements of our samples, conducted in a commercial superconducting quantum interference device magnetometer (Quantum Design Magnetic Properties Measurement System) on nonmicrostructured reference samples, reveal that in the as-prepared state, the $\mathrm{Cu}_{2} \mathrm{MnAl}$ layers are nonferromagnetic (Fig. 1) as typical for Heusler layers prepared at room temperature. ${ }^{17}$ Only after annealing at $240{ }^{\circ} \mathrm{C}$ for $24 \mathrm{~h}$ a ferromagnetic hysteresis loop develops (see inset of Fig. 1). In the as-prepared state the unit cell of the $\mathrm{Cu}_{2} \mathrm{MnAl}$ layers has $\mathrm{A} 2$ symmetry with a 


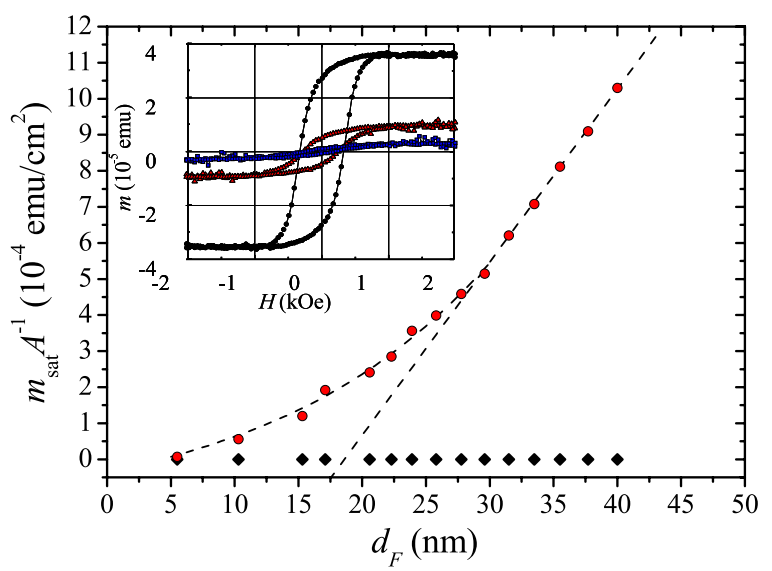

FIG. 1. (Color online) Magnetic moment (measured at $1 \mathrm{kOe}$ and $15 \mathrm{~K}$ ) divided by the sample area versus the thickness of the Heusler layer in the as-prepared state (diamonds) and after annealing at $240{ }^{\circ} \mathrm{C}$ for $24 \mathrm{~h}$ (dots). The dashed linear slope corresponds to a magnetic moment of $2.9 \mu_{B} / \mathrm{Mn}$ atom. The inset shows hysteresis loops measured at $15 \mathrm{~K}$ for samples in the annealed state with the Heusler layer thickness $d_{F}=5.4 \mathrm{~nm}$ (inner loop), $d_{F}$ $=10.2 \mathrm{~nm}$ (middle loop), and $d_{F}=17 \mathrm{~nm}$ (outer loop).

random distribution of all atoms on a bcc lattice. ${ }^{17}$ In this structure nearest-neighbor $\mathrm{Mn}$ atoms are coupled by strong antiferromagnetic exchange interactions whereas Mn nextnearest neighbors with an $\mathrm{Al}$ atom in between are coupled ferromagnetically via an indirect type of exchange interaction. ${ }^{18}$ The competition of these two interactions and the randomness of the atomic distribution in the A2 structure gives rise to spin-glass magnetic order with a very low value for the magnetization. Upon annealing at $240{ }^{\circ} \mathrm{C}$ the unit cell symmetry transforms toward the ordered L $2{ }_{1}$-type Heusler structure which is a combination of four interpenetrating fcc sublattices occupied by $\mathrm{Mn}, \mathrm{Cu}$, and $\mathrm{Al}$, exclusively. ${ }^{17}$ With $\mathrm{L} 2_{1}$ symmetry in $\mathrm{Cu}_{2} \mathrm{MnAl}$ there are no $\mathrm{Mn}-\mathrm{Mn}$ nearest neighbors and the ferromagnetic Mn-Al-Mn exchange interaction leads to strong ferromagnetic order with a magnetic moment of about 3.2 $\mu_{\mathrm{B}} / \mathrm{Mn}$ atom and a ferromagnetic $\mathrm{Cu}-$ rie temperature of $603 \mathrm{~K} \cdot{ }^{17}$

In the annealed state a ferromagnetic hysteresis loop is observed above a critical thickness of the Heusler layers $d_{F}=5 \mathrm{~nm}$, for $d_{F}<5 \mathrm{~nm}$ there is no ferromagnetic magnetization, indicating that at both interfaces of the Heusler layers an interlayer of $2.5 \mathrm{~nm}$ thickness with spin-glass order persists. Above $d_{F}=5 \mathrm{~nm}$ the saturation magnetic moment per Mn spin increases gradually over a very broad thickness range (Fig. 1). The microscopic origin of this behavior is an intrinsic gradient of the degree of L 2 -type atomic order inside the Heusler layers with a low degree of order at the interfaces and a higher degree of order in the interior of the film. ${ }^{19}$

The Josephson critical current of the junctions is determined by taking the $I-V$ characteristics for different in-plane magnetic fields. We observe textbooklike Fraunhofer patterns (Fig. 2) for both, the as-prepared and the annealed states with the critical current vanishing at the minima thus giving clear evidence of a high quality and homogeneity of the $\mathrm{AlO}_{x}$-as well as the $\mathrm{Cu}_{2} \mathrm{MnAl}$ barrier.

The thickness dependence of the critical current density in the as-prepared state is plotted in Fig. 3. One observes an exponentially damped curve with a decay length $\xi=0.8 \mathrm{~nm}$, as typical for a system with strong pair-breaking scattering. Strong pair-breaking scattering in the Heusler layers must be expected since in the spin-glass state there is a high density of randomly oriented $\mathrm{Mn}$ magnetic moments. The decay length of a dirty metal $\left(\ell_{m} \ll \xi_{F}\right)$ with pair-breaking scattering $\left(E_{i e} \gg k_{B} T\right)$ is given by $\xi_{N}=\sqrt{\hbar D_{F} / E_{i e}}$ with the diffusion constant $D_{F}$ and the scattering energy $E_{i e}=\hbar / \tau_{i e}$ (scattering time $\tau_{i e}$ ). Single $\mathrm{Cu}_{2} \mathrm{MnAl}$ layers in the as-prepared state have a residual resistivity of $\rho_{m} \approx 275 \mu \Omega \mathrm{cm}$ and with the Fermi velocity $v_{F}$ taken from the literature ${ }^{20}$ we can estimate $D_{F}$ and thus derive $E_{i e}=45 \mathrm{meV}$.

The thickness dependence for the same Josephson junctions after annealing has also been plotted in Fig. 3. In the thickness range below $d_{F} \approx 7 \mathrm{~nm}$ the critical current in the annealed state is smaller than in the as-prepared state by a constant factor of about 0.6 , this is mainly caused by an increase in the $\mathrm{AlO}_{x}$ barrier resistance $R_{n}$ by about $40 \%$ upon annealing. Above $d_{F}=7 \mathrm{~nm}$ the two curves $j_{c}\left(d_{F}\right)$ in Fig. 3, cross and in the annealed state the critical current is enhanced (see also Fig. 2). The enhancement factor reaches a maximum value of nearly 100 close to $d_{F}=10.5 \mathrm{~nm}$. This is surprising since for the ferromagnetic state of the $\mathrm{Cu}_{2} \mathrm{MnAl}$ layers we had expected a suppression of the critical current rather than an enhancement. Above the plateau in the critical current, at $d_{F} \approx 10.5 \mathrm{~nm}$ we find an additional remarkable feature: there is a sharp decay and within a thickness range of $0.3 \mathrm{~nm}$ the critical current drops below the experimental resolution limit.

Examples for the temperature dependence of the critical current are shown in Fig. 4. For all samples in the asprepared state and for the samples in the annealed state out-
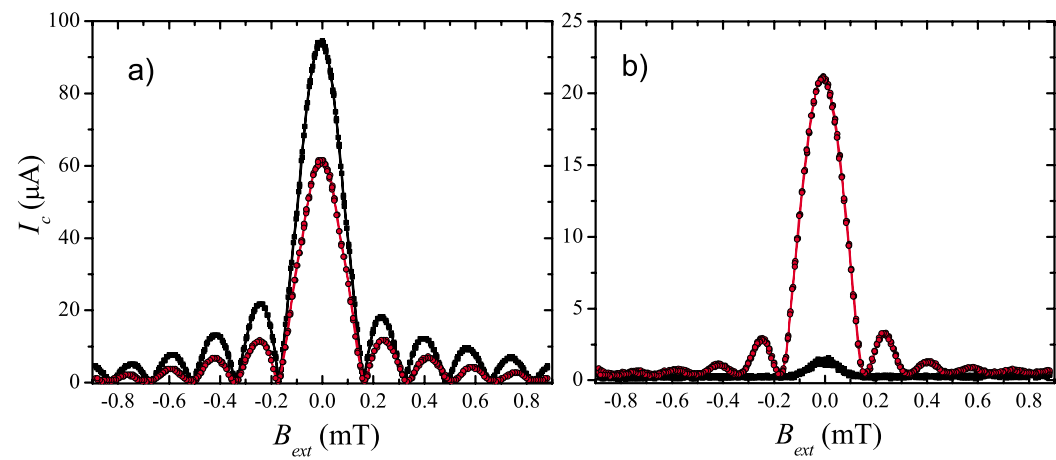

FIG. 2. (Color online) Critical current versus the applied magnetic field for two junctions $\left(10 \times 50 \mu \mathrm{m}^{2}\right)$ with a thickness of the Heusler layer (a) $d_{F}=6.7 \mathrm{~nm}$ and (b) $d_{F}=9.3 \mathrm{~nm}$ in the as-prepared state (black squares) and the annealed state (red circles). 


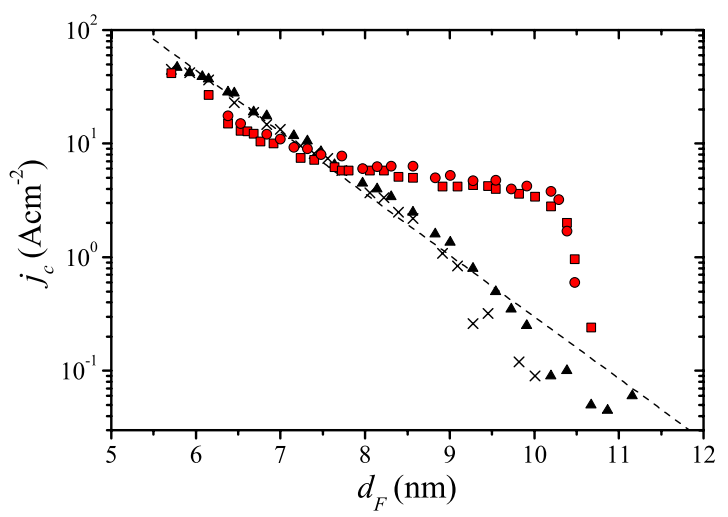

FIG. 3. (Color online) Critical current density (log) versus the Heusler layer thickness in the as-prepared state (crosses: $10 \times 50 \mu \mathrm{m}^{2}$ and triangles: $10 \times 200 \mu \mathrm{m}^{2}$ ) and the annealed state (circles: $10 \times 50 \mu \mathrm{m}^{2}$ and squares: $10 \times 200 \mu \mathrm{m}^{2}$ ). Measurement done at $4.2 \mathrm{~K}$. The dashed line corresponds to a damping length $\xi=0.8 \mathrm{~nm}$.

side the plateau region in Fig. 3 we find a monotonous increase in the critical current with decreasing temperature, as in conventional Josephson junctions. Within the plateau region we observe an anomalous, noncontinuous behavior with a broad maximum in $j_{c}(T)$ at about $4.5 \mathrm{~K}$ and $j_{c}$ decreasing toward lower temperatures.

Before invoking unconventional superconductivity for the interpretation of the results, we first try to interpret them in terms of conventional singlet superconductivity. The theory for singlet pairing of junctions with a ferromagnetic barrier of variable thickness $d_{F}$ in the dirty limit predicts the functional dependence,

$$
j_{c}\left(d_{F}\right)=j_{0} \exp \left(-\frac{d_{F}}{\xi_{F 1}}\right)\left|\cos \left(\frac{d_{F}}{\xi_{F 2}}\right)\right|
$$

with the decay length $\xi_{F 1}$ and the oscillation length $\xi_{F 2} .5$ The second term in Eq. (1) describes the Josephson phase transition between a 0 - and $\pi$-type junctions and leads to a deviation of the current density from an exponentially damped curve toward smaller values with increasing thickness, in sharp contrast to the plateau in $j_{c}\left(d_{F}\right)$ observed for the annealed state in Fig. 3. For singlet superconductivity the formation of the plateau could be explained by a decay length $\xi_{F 1}$ continuously increasing with increasing thickness $d_{F}$. Principally this is possible, if an increasing diffusion constant $D_{F}$ and a decreasing pair-breaking scattering rate $1 / \tau_{\text {ie }}$ overcompensates the increasing ferromagnetic exchange energy $E_{e x}$. Since we have no direct experimental access to these parameters, we cannot rule out this possibility. However, within this model it is difficult to explain why the onset of the plateau at $d_{F} \approx 7 \mathrm{~nm}$ does not coincide with the onset of ferromagnetism at $d_{F} \approx 5 \mathrm{~nm}$. Furthermore, the most striking feature in $j_{c}\left(d_{F}\right)$, namely, the sharp drop-off of the critical current between $d_{F}=10.3 \mathrm{~nm}$ and $d_{F}=10.6 \mathrm{~nm}$ is difficult to reconcile with singlet pairing. In this thickness range the ferromagnetic saturation moment increases only slightly by about $5 \%$ and we find no plausible reason for a drastic change in the ferromagnetic exchange energy $E_{e x}$.

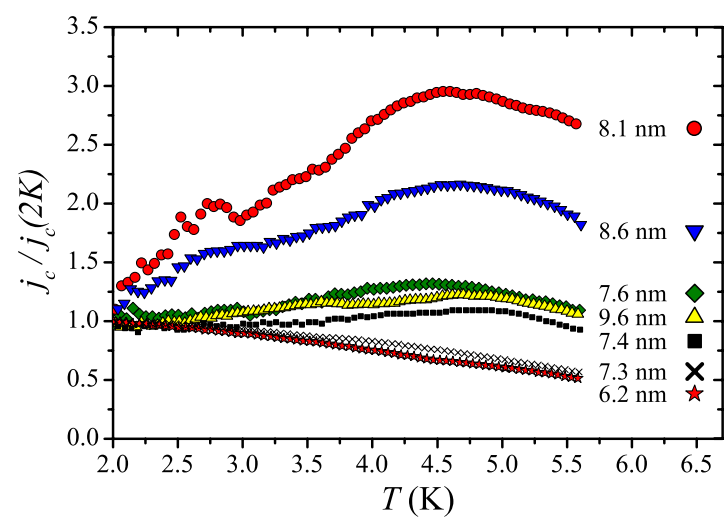

FIG. 4. (Color online) Normalized critical current density versus temperature for junctions with different Heusler thicknesses (as prepared: $d_{F}=7.3 \mathrm{~nm}$, annealed: all others, $A=10 \times 50 \mu \mathrm{m}^{2}$ ).

Last not least, we find that the anomalous temperature dependence of the critical current in the range of the plateau is difficult to explain. A positive temperature coefficient $j_{c}(T)$ can occur close to $0-\pi$ transition, ${ }^{21}$ but only if the thermal energy $k_{B} T$ is of the same order as $E_{i e}+E_{e x}$, which we can exclude here.

The alternative interpretation for the anomalous thickness dependence in Fig. 3 is the assumption of an additional component of the supercurrent which compensates the exponentially damped singlet supercurrent. The theoretical work ${ }^{5,6}$ strongly suggests that this additional component should be identified as an odd-frequency triplet supercurrent. This assumption implies that inside the Heusler layers there must be a mechanism which very effectively generates triplet superconductivity. In Fig. 1 one sees that the anomalous feature occurs in a relatively narrow thickness range only, thus the microscopic origin for the conversion of the singlet into triplet pairs seems to depend sensitively on the magnetization profile inside the Heusler layers. A similar sensitivity of the triplet component to the thickness of the interface layers has also been observed in Ref. 15 .

Although we have no direct experimental information about the specific magnetization profile inside the $\mathrm{Cu}_{2} \mathrm{MnAl}$ layers in our junctions, from our knowledge of the magnetization profile inside other Heusler layer systems ${ }^{19}$ we suggest the following interpretation for the relation between the development of the magnetization profile and the appearance of the triplet component in the supercurrent: in the thickness range just above the onset of ferromagnetism, ferromagnetic order already exists in the core of the Heusler layers, whereas at the interfaces spin-glass order still prevails. The coupling between the two types of magnetic order will induce a small ferromagnetic magnetization in the interface layers, however, with some canting of the local magnetization from the direction of the core magnetization because of the coupling to the coexisting random spin-glass type of order. It is appealing to identify these canted interface moments as the "spin active zone" needed for the conversion of singlet pairs into triplet pairs. ${ }^{12,13}$ With increasing thickness and concomitantly increasing strength of the ferromagnetic order in the core of the Heusler layers, the canting angle will decrease and the spin active zones will be eliminated. This 
defines the upper thickness limit of the plateau in $j_{c}\left(d_{F}\right)$. At the lower thickness boundary of the plateau the spin active interface layers and the ferromagnetic core layer first must be formed, therefore this boundary is shifted by $2 \mathrm{~nm}$ compared to the onset of ferromagnetism. This model for the generation of triplet superconductivity in the $\mathrm{Cu}_{2} \mathrm{MnAl}$ layers has very recently been nicely reproduced by theoretical calculations modeling our system very closely. ${ }^{22}$ Within the model of odd-triplet superconductivity we also find a reasonable interpretation for the anomalous temperature dependence as resulting from the competition of different contributions to the supercurrent in junctions with unconventional pairing. ${ }^{23}$

In summary, we have shown that high-quality Josephson junctions with $\mathrm{Cu}_{2} \mathrm{MnAl}-\mathrm{Heusler}$ barriers can be prepared. The Heusler barriers offer the opportunity to change the magnetic state of the barrier by annealing the complete junc- tions. The transformation of the magnetic state of the Heusler layers from the spin glass to the ferromagnetic state is accompanied by an enhancement of the supercurrent density by up to two orders of magnitude. We argued that the anomalous behavior of the supercurrent is difficult to reconcile with conventional singlet pairing and proposed an interpretation in terms of an odd-triplet contribution to the supercurrent. However, an unambiguous proof of the triplet character of the supercurrent is not provided by our present experimental results and must wait for future experimental verification.

The authors thank the DFG for funding this work within the DFG under Project No. WE 4359/1-1 and within the SFB 491. Additionally we thank A. F. Volkov, M. Fistoul, and K. B. Efetov for valuable discussions.
${ }^{1}$ K. Balian and R. P. Werthamer, Phys. Rev. 131, 1553 (1963).

${ }^{2}$ D. Aoki, A. Huxley, E. Ressouche, D. Braithwaite, J. Flouquet, J.-P. Brison, E. Lhotel, and C. Paulsen, Nature (London) 413, 613 (2001).

${ }^{3}$ C. C. Tsuei and J. R. Kirtley, Rev. Mod. Phys. 72, 969 (2000).

${ }^{4}$ A. P. Mackenzie and Y. Maeno, Rev. Mod. Phys. 75, 657 (2003).

${ }^{5}$ A. I. Buzdin, Rev. Mod. Phys. 77, 935 (2005).

${ }^{6}$ F. S. Bergeret, A. F. Volkov, and K. B. Efetov, Rev. Mod. Phys. 77, 1321 (2005).

${ }^{7}$ R. S. Keizer, S. T. B. Goennewein, T. M. Klapwijk, G. Miao, G. Xiao, and A. Gupta, Nature (London) 439, 825 (2006).

${ }^{8}$ I. Sosnin, H. Cho, V. T. Petrashov, and A. F. Volkov, Phys. Rev. Lett. 96, 157002 (2006).

${ }^{9}$ T. Kontos, M. Aprili, J. Lesueur, F. Genet, B. Stephanidis, and R. Boursier, Phys. Rev. Lett. 89, 137007 (2002); J. W. A. Robinson, S. Piano, G. Burnell, C. Bell, and M. G. Blamire, Phys. Rev. B 76, 094522 (2007); V. A. Oboznov, V. V. Bol'ginov, A. K. Feofanov, V. V. Ryazanov, and A. I. Buzdin, Phys. Rev. Lett. 96, 197003 (2006).

${ }^{10}$ F. S. Bergeret, A. F. Volkov, and K. B. Efetov, Phys. Rev. Lett. 86, 4096 (2001).

${ }^{11}$ F. S. Bergeret, A. F. Volkov, and K. B. Efetov, Phys. Rev. B 68 , 064513 (2003).

${ }^{12}$ M. Houzet and A. I. Buzdin, Phys. Rev. B 76, 060504(R) (2007).

${ }^{13}$ M. Eschrig, J. Kopu, J. C. Cuevas, and G. Schön, Phys. Rev. Lett. 90, 137003 (2003).

${ }^{14}$ V. L. Berezinskii and C. Baraduc, JETP Lett. 20, 287 (1974).
${ }^{15}$ T. S. Khaire, M. A. Khasawneh, W. P. Pratt, Jr., and N. O. Birge, Phys. Rev. Lett. 104, 137002 (2010).

${ }^{16} \mathrm{M}$. Weides, K. Tillmann, and H. Kohlstedt, Physica C 437-438, 349 (2006); M. Weides, M. Kemmler, E. Goldobin, D. Koelle, R. Kleiner, H. Kohlstedt, and A. Buzdin, Appl. Phys. Lett. 89, 122511 (2006); M. Weides, M. Kemmler, H. Kohlstedt, R. Waser, D. Koelle, R. Kleiner, and E. Goldobin, Phys. Rev. Lett. 97, 247001 (2006).

${ }^{17}$ P. J. Webster and K. R. A. Ziebeck, Heusler Alloys, LandoltBörnstein New Series, Group III Vol. 19, Pt. C (Springer, Berlin, 1986); S. Picozzi, A. Continenza, and A. J. Freeman, Phys. Rev. B 69, 094423 (2004); I. Galanakis, P. H. Dederichs, and N. Papanikolaou, ibid. 66, 134428 (2002).

${ }^{18}$ J. Kübler, A. R. Williams, and C. B. Sommers, Phys. Rev. B 28 , 1745 (1983).

${ }^{19}$ J. Grabis, A. Bergmann, A. Nefedov, K. Westerholt, and H. Zabel, Phys. Rev. B 72, 024438 (2005); A. Bergmann, J. Grabis, B. P. Toperverg, V. Leiner, M. Wolff, H. Zabel, and K. Westerholt, ibid. 72, 214403 (2005).

${ }^{20}$ N. G. Fenander, L. Wiktorin, and H. P. Myres, J. Phys. Chem. Solids 29, 1973 (1968).

${ }^{21}$ V. V. Ryazanov, V. A. Oboznov, A. Yu. Rusanov, A. V. Veretennikov, A. A. Golubov, and J. Aarts, Phys. Rev. Lett. 86, 2427 (2001).

22 J. Linder and A. Sudbo, Phys. Rev. B 82, 020512 (2010).

${ }^{23}$ M. Eschrig, T. Löfwander, T. Champel, J. C. Cuevas, J. Kopu, and G. Schön, J. Low Temp. Phys. 147, 457 (2007). 